\title{
Acquired haemophilia A in an elderly patient related to relapsed cervix carcinoma
}

Fatos Dilan Atilla, Ahmet Alp Unat, Hale Bulbul, Murat Ulukus, Zuhal Demirci, Guray Saydam, Fahri Sahin

Acquired haemophilia $\mathrm{A}(\mathrm{AHA})$ is a rare, autoimmune disease, presenting as sudden haemorrhages without any personal or family history. Anti-factor VIII (FVIII) is the most commonly recognised autoantibody resulting in decreased factor activity. The aetiology and pathophysiology of these antibodies remains unclear. Approximately $50 \%$ of reported cases are idiopathic; the rest are associated with other conditions, mainly underlying malignancies, autoimmune diseases (eg rheumatoid arthritis (RA), systemic lupus erythematosus), drug administration and postpartum

FATOS DILAN ATILLA

Adult Haemophilia and Thrombosis Centre, Ege University Hospital, Izmir, Turkey. Email: fatosdilanatilla@gmail.com

AHMET ALP UNAT

Department of Internal Medicine, Ege University Hospital, Izmir, Turkey

HALE BULBUL

Adult Haemophilia and Thrombosis Centre, Ege University Hospital, Izmir, Turkey

\section{MURAT ULUKUS}

Department of Obstetrics and Gynaecology, Ege

University Hospital, Izmir, Turkey

ZUHAL DEMIRCI

Adult Haemophilia and Thrombosis Centre, Ege University Hospital, Izmir, Turkey

GURAY SAYDAM

Adult Haemophilia and Thrombosis Centre, Ege University Hospital, Izmir, Turkey

FAHRI SAHIN

Adult Haemophilia and Thrombosis Centre, Ege University Hospital, Izmir, Turkey

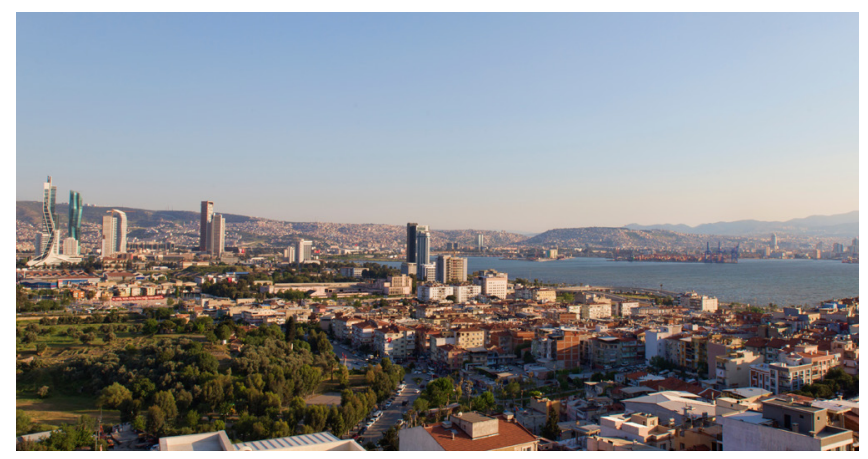

Immunosuppresive treatment of an elderly patient with acquired haemophilia A at Ege University Hospital was potentially complicated by existing corticosteroid treatment for rheumatoid arthritis. However, she responded well to first-line therapy and the underlying cancer it related to was not contraindicated

period. A 74-year-old woman presented to our institution with haematochezia and haematuria. She had a medical history of cervix carcinoma; total abdominal hysterectomy and bilateral salpingooophorectomy with postoperative chemo-radiotherapy was performed in 2011. She had also been followed up for 20 years for deforming and severe RA, which was in low-disease activity with methotrexate and corticosteroid. Laboratory investigations for abnormal bleeding revealed prolongation of activated partial thromboplastin time (APTT). APTT prolongation was not corrected by 50:50 plasma mixing studies, and a confirmatory factor assay demonstrated FVIII deficiency (1.4 IU/dL; normal range 50-150 IU/dL). Positive FVIII antibodies on Bethesda testing confirmed a diagnosis of AHA. A rectosigmoid mass and fistula between rectum and bladder were discovered by computed tomography

This is an Open Access article distributed under the terms of the Creative Commons Attribution-NonCommercial-NoDerivs License (https://creativecommons.org/licenses/by-nc-nd/3.0/) which permits use and distribution in any medium, provided the original work is properly cited, the use is non-commercial, and no modifications or adaptations are made. Copyright is retained by the authors. 
(CT). Bleeding was controlled with recombinant activated factor VII ( $\mathrm{rFVIla}$ ) after two weeks. Eradication of the inhibitor was achieved with high-dose pulse methylprednisolone for two days and then $2 \mathrm{mg} / \mathrm{kg}$ daily over four weeks.

Keywords: Acquired haemophilia A, bleeding, cancer, rheumatoid arthritis

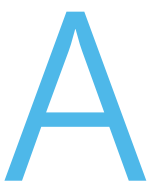

cquired haemophilia $A(A H A)$ is a rare autoimmune disease caused by the development of an autoantibody against

factor VIII (FVIII). It presents without any personal or family history, with the most common bleeding sites being the skin or mucous membranes, muscles and soft tissue [1]. In contrast to patients with congenital haemophilia, haemarthroses are rarely seen in AHA patients; haemorrhages often develop spontaneously, and can also result from minor trauma and iatrogenic injuries. Anti-factor VIII autoantibodies are mostly IgG-structured; their action against FVIII results in decreased factor activity [2]. The aetiology and pathophysiology of these antibodies remains unclear. Approximately $50 \%$ of reported cases are idiopathic; the remainder are associated with other conditions, mainly underlying malignancies, autoimmune diseases such as rheumatoid arthritis or systemic lupus erythematosus, drug administration and the postpartum period [3]. The occurrence of these autoantibodies is rare, with a rate of approximately one person per million each year, with an average age of 75 years and without any sex predominance [4]. Overall, FVIII inhibitors affect both sexes equally. Severe or life-threatening bleeding occurs in more than $80 \%$ of patients. The mortality rate has been estimated to be in the range of $7.9 \%$ to $22 \%$, with most haemorrhagic deaths occurring within the first few weeks after presentation $[1,6]$. The reduction in the mortality rate observed in the most recent studies may reflect therapeutic improvements in the management of acute bleeding during the last few years (first porcine FVIII and activated prothrombin complex concentrate (aPCC), and then recombinant activated factor VII (rFVIIa)) [7].

\section{CASE PRESENTATION}

A 74-year-old Caucasian woman presented to Ege University Hospital in Izmir, Turkey, with heavy bleeding related to haematochezia and haematuria, which were complicated by severe anaemia. She had a medical history of cervix carcinoma; total abdominal hysterectomy and bilateral salpingo-oophorectomy with postoperative chemo-radiotherapy were performed in 2011. She also had deforming and severe rheumatoid arthritis (RA) with positive rheumatic serum (positive for rheumatoid factor and anti-cyclic citrullinated peptide).

Laboratory investigations performed for abnormal bleeding revealed prolongation of activated partial thromboplastin time (APTT). APTT prolongation was not corrected by 50:50 plasma mixing studies, and confirmatory factor assay demonstrated FVIII deficiency (1.4 IU/dL; normal range 50-150 IU/dL). Positive FVIII antibodies (117 BU/mL) on Bethesda testing confirmed a diagnosis of AHA. Prothrombin time (PT) and international normalised ratio (INR) were both in the normal range. Platelet and leukocyte levels were normal in the blood count, which nevertheless revealed hypochromic and microcytic anaemia at $6.4 \mathrm{~g} / \mathrm{dL}$ of haemoglobin $(\mathrm{Hb})$, originating from an iron deficiency.

Computed tomography (CT) showed a rectosigmoid mass and rectovesical fistula, although there was no evidence of internal bleeding. A three-way urethral catheter was placed and continuous irrigation was performed by a consultant urologist. A bypassing agent was administered; the patient received 3mg (70-90 $\mathrm{mcg} / \mathrm{kg}$ ) rFVlla every four hours for the first two days, then every six hours for four days, and every 12 hours for six days. Bleeding, Hb level and APTT were followed up daily. Four units of packed red blood cells were transfused over a two-week period.

The patient had been followed up for a destructive rheumatoid arthritis for 20 years, with no systemic impairment. Her arthritis was in low-disease activity with $10 \mathrm{mg}$ methotrexate weekly and $8 \mathrm{mg}$ of methylprednisolone per day. Although corticosteroids are the first line of immunosuppressive treatment for autoantibody eradication, the fact that the patient had been on corticosteroid therapy over a long period of time meant that immunomodulatory therapy could be complicated. Nevertheless, high-dose methylprednisolone was given for immunosuppression with the aim of eradicating the autoantibody or suppressing the cell clone responsible for its synthesis. $500 \mathrm{mg}$ pulse methylprednisolone parenteral was administered for two days to achieve a satisfactory immunosuppressive effect. Thereafter $2 \mathrm{mg} / \mathrm{kg}$ per day methylprednisolone was initiated, which was gradually tapered over four weeks. At the same time, the patient continued to receive methotrexate at $10 \mathrm{mg}$ per week.

After four weeks of treatment with methylprednisolone, FVIII was $66 \%$ and the anti-FVIII 


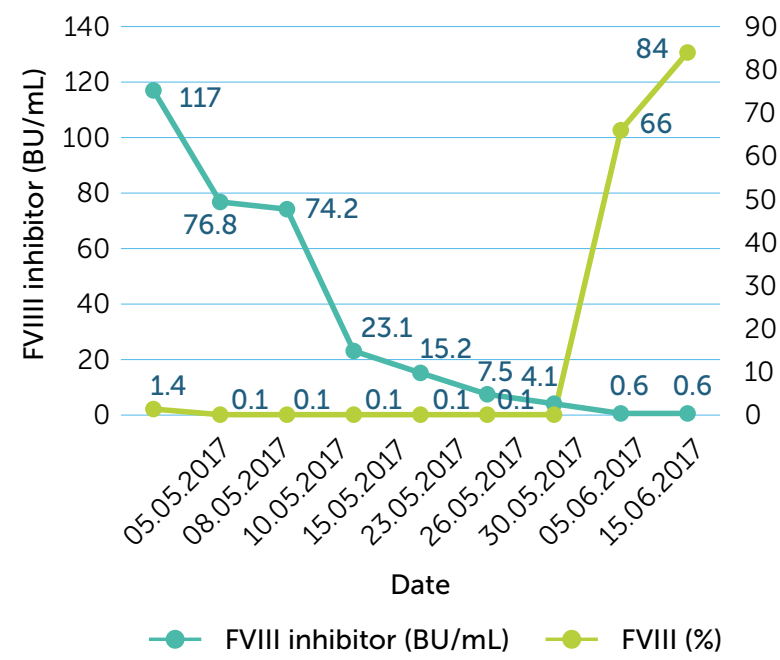

Figure 1: Response levels of FVIII and FVIII inhibitor treated with methylprednisolone

inhibitor was negative $(<0.6 \mathrm{BU} / \mathrm{mL})$. FVIII and FVIII inhibitor levels were followed intermittently. Response of the levels of FVIII-FVIII inhibitor and APTT-Hb are shown in Figure 1 and 2 respectively.

Bleeding was controlled with rFVIla 3mg (70-90 $\mathrm{mcg} / \mathrm{kg}$ ) every four hours for the first two days, every six hours for four days and then every twelve hours for six days. Haematochezia and haematuria were settled but ongoing for two weeks. By the end of the second week, bleeding had stopped and rFVIla was withdrawn.

Biopsy was performed from the rectosigmoid mass when APTT and FVIII had normalised. After histomorphologic evaluation, cervix adenocarcinoma was revealed. Palliative chemotherapy was considered. However, two days after discharge from the ward, the patient was admitted to emergency service with acute abdominal pain and died suddenly due to perforation of the descending colon.

\section{DISCUSSION}

$\mathrm{AHA}$ is a rare bleeding diathesis caused by autoantibodies directed against clotting FVIII and associated with an increased morbidity and mortality. In approximately 50\% of cases, FVIII autoantibodies occur in patients lacking relevant concomitant diseases ${ }^{[8]}$. Nearly $10 \%$ of patients with AHA have an underlying malignancy, either solid or haematologic ${ }^{[7,9]}$. AHA is also associated autoimmune disease; $4-8 \%$ of cases are associated with rheumatoid arthritis ${ }^{[10]}$. Patients with $\mathrm{AHA}$ are predisposed to developing antibodies and high-titre inhibitors, meaning that intense treatment is often required ${ }^{[7]}$.

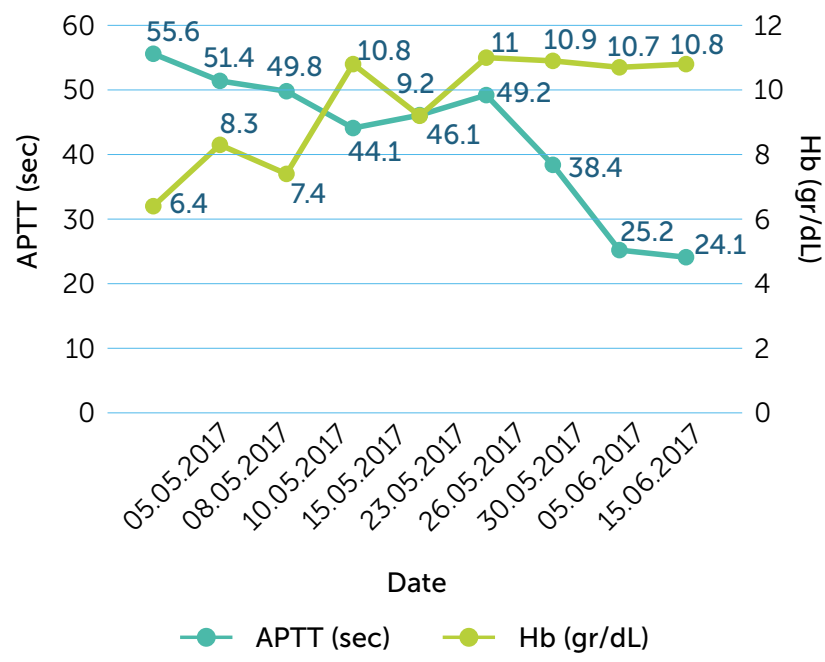

Figure 2: Haemoglobin concentration and APTT level during treatment are shown (four units of packed red blood cells were transfused at timeline)

The case presented above involved both rheumatoid arthritis and relapsed cervix carcinoma. FVIII autoantibodies were eradicated with high-dose methylprednisolone without the need for second-line immunosuppressive treatment. The aetiology of AHA was not precisely manifested, but a good response to corticosteroid suggested that it was related to the relapsed cervix carcinoma. In addition, AHA became evident concurrent with relapsing symptoms of cervix carcinoma, further indicating its relevance.

AHA has quite different pathophysiology, clinic presentation and evaluation compared to hereditary haemophilia. The diagnosis of AHA in a patient with no previous personal or family history of bleeding is typically based on the initial detection of an isolated prolongation of APTT, which cannot be corrected by incubating equal volumes of patient plasma and normal plasma for two hours at $37^{\circ} \mathrm{C}$ (mixing study). Identification of a reduced FVIII level with evidence of FVIII inhibitor activity (titrated using the Bethesda assay or its Nijmegen modification) is the key point to reach the appropriate diagnose ${ }^{[4]}$. However, assaying FVIII inhibitor activity is not easily accessible in many health institutions in Turkey, meaning that a mixing study is mandatory in making the initial diagnosis.

The treatment of AHA has two main goals: to arrest bleeding and to eradicate the inhibitor. The first objective is necessary because bleeding episodes are usually severe and can be life-threatening; the second is required to restore normal haemostasis and may be accomplished using immunotherapy. Patients with mild 
bleeding could be treated with desmopressin (DDVAP) at a dose of $0.3 \mathrm{mcg} / \mathrm{kg}$ daily for three to five days. If the bleeding is severe and inhibitor levels are low (inhibitor titres under $5 \mathrm{BU} / \mathrm{mL}$ ), large doses of plasma-derived or recombinant human FVIII concentrates may be reasonable. However, the dose of human FVIII needed to neutralise a circulating inhibitor to provide FVIII haemostatic levels would be very high ${ }^{[2]}$. If the inhibitor levels are higher than $5 \mathrm{BU} / \mathrm{mL}$, human FVIII product would be ineffective. Bypassing agents are currently the most used first-line treatment, and both rFVIla and $\mathrm{APCC}$ have proven effective in the treatment of $\mathrm{AHA}^{[11,12]}$. The recommended dose of aPCC is $50-200$ IU/kg/d in divided doses. Larger doses (>200 IU/kg/d) have been associated with adverse thrombotic events ${ }^{[12]}$. The recommended dose of $\mathrm{rFVIIa}$ is an intravenous bolus dose of 90 to $120 \mu \mathrm{g} / \mathrm{kg}$ repeated every two to three hours, depending on the clinical response ${ }^{[11,13]}$.

Patients with cancer-associated FVIII inhibitors, including patients with solid and hematologic malignancies, have achieved $70 \%$ complete responses rate to treatment ${ }^{[9]}$. The presence of an underlying cancer is not a contraindication to the use of immunosuppressive therapy; these patients should be treated in the same manner as other patients with AHA.

AHA should be considered in the differential diagnosis of patients with spontaneous haemorrhage and isolated prolongation of APTT. It is important that action is taken immediately in order to facilitate a quick and definitive diagnose in patients with severe or lifethreatening bleeding.

\section{ACKNOWLEDGEMENTS}

The authors have advised no interests that might be perceived as posing a conflict or bias.

Informed consent has been obtained from the guardian of the patient reported in this case study.

\section{REFERENCES}

1. Green D, Lechner K. A survey of 215 non-hemophilic patients with inhibitors to Factor VIII. Thromb Haemost 1981; 45(3): 200-3.

2. Cohen AJ, Kessler CM. Acquired inhibitors. Baillieres Clin Haematol 1996; 9(2): 331-54.

3. Knoebl P, Marco P, Baudo F, et al. Demographic and clinical data in acquired hemophilia $A$ : results from the European Acquired Haemophilia Registry (EACH2). J Thromb Haemost 2012; 10(4): 622-31.

4. Delgado J, Jimenez-Yuste V, Hernandez-Navarro F, Villar A. Acquired haemophilia: review and meta-analysis focused on therapy and prognostic factors. Br J Haematol 2003; 121(1): 21-35.

5. Bouvry P, Recloux P. Acquired hemophilia. Haematologica 1994; 79(6): 550-6.

6. Yee TT, Taher A, Pasi KJ, Lee CA. A survey of patients with acquired haemophilia in a haemophilia centre over a 28-year period. Clin Lab Haematol 2000; 22(5): 275-8.

7. Franchini M, Gandini G, Di Paolantonio T, Mariani G. Acquired hemophilia A: a concise review. Am J Hematol 2005; 80(1): 55-63.

8. Hay CR. Acquired haemophilia. Baillieres Clin Haematol 1998; 11(2): 287-303

9. Sallah S, Wan JY. Inhibitors against factor VIII in patients with cancer. Analysis of 41 patients. Cancer 2001; 91(6): 1067-74.

10. O'Connor CR. Systematic review of the presentation of coagulation factor VIII inhibitors in rheumatic diseases: A potential cause of life-threatening hemorrhage. Semin Arthritis Rheum 2015; 44(6): 695-709.

11. Hay CR, Negrier C, Ludlam CA. The treatment of bleeding in acquired haemophilia with recombinant factor VIla: a multicentre study. Thromb Haemost 1997; 78(6): 1463-7.

12. Lusher JM. Use of prothrombin complex concentrates in management of bleeding in hemophiliacs with inhibitors-benefits and limitations. Semin Hematol 1994; 31(2 Suppl 4): 49-52.

13. Giangrande P. Acquired hemophilia. Revised edition. Treatment of hemophilia, no. 38. World Federation of Hemophilia, 2012. Available from http://www1.wfh.org/publication/files/pdf1186.pdf (accessed 25 November 2018).

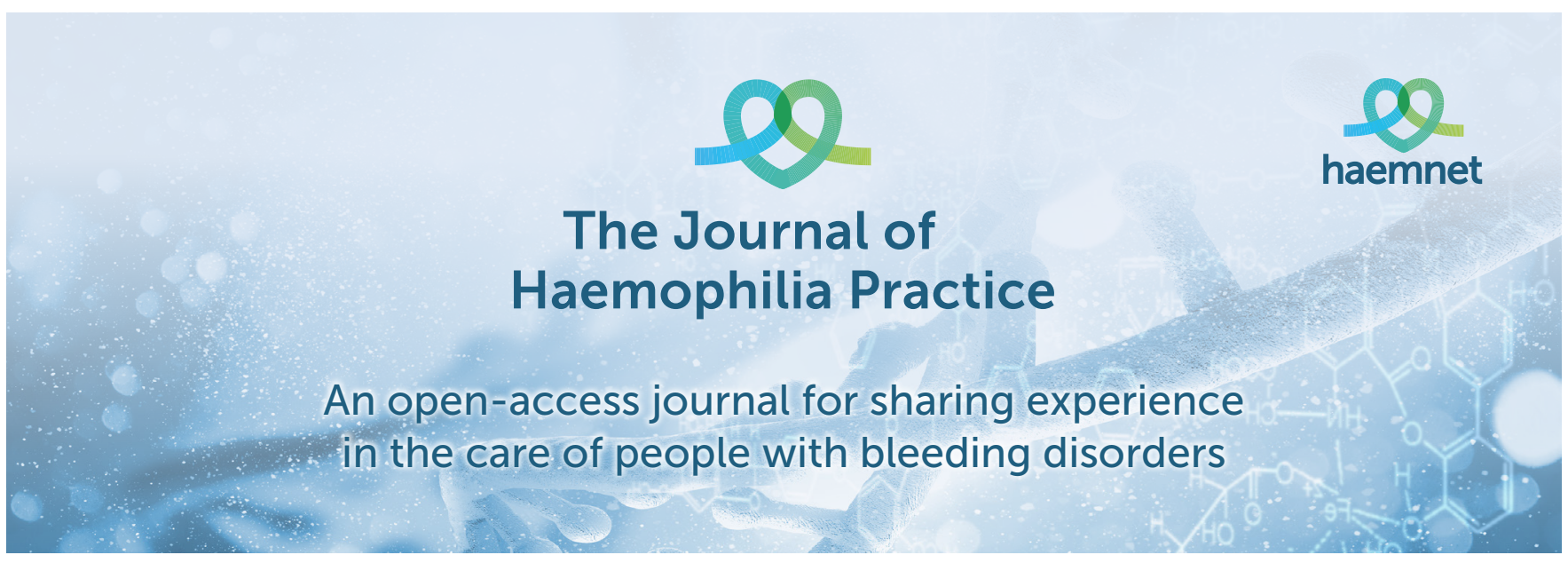

